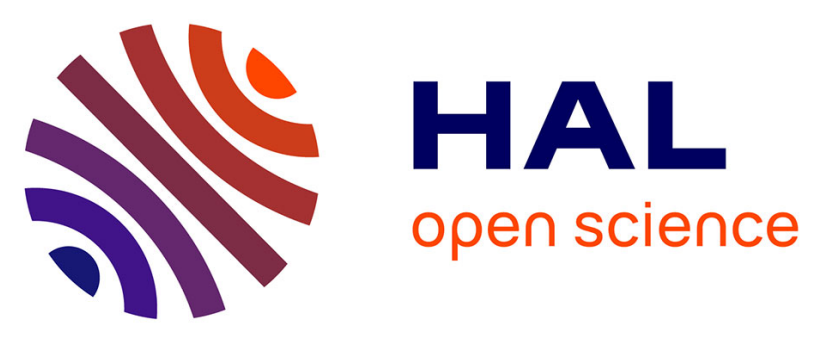

\title{
Study of the radiated polarization of an antenna array with circular geometry
}

Karim Louertani, Régis Guinvarc'H, Nicolas Ribière-Tharaud, Marc Hélier

\section{To cite this version:}

Karim Louertani, Régis Guinvarc'H, Nicolas Ribière-Tharaud, Marc Hélier. Study of the radiated polarization of an antenna array with circular geometry. Progress In Electromagnetics Research C, 2011, 24, pp.173-183. 10.2528/PIERC11061706 . hal-00629215

\section{HAL Id: hal-00629215 https://hal.sorbonne-universite.fr/hal-00629215}

Submitted on 30 Jul 2021

HAL is a multi-disciplinary open access archive for the deposit and dissemination of scientific research documents, whether they are published or not. The documents may come from teaching and research institutions in France or abroad, or from public or private research centers.
L'archive ouverte pluridisciplinaire HAL, est destinée au dépôt et à la diffusion de documents scientifiques de niveau recherche, publiés ou non, émanant des établissements d'enseignement et de recherche français ou étrangers, des laboratoires publics ou privés. 


\title{
STUDY OF THE RADIATED POLARIZATION OF AN ANTENNA ARRAY WITH CIRCULAR GEOMETRY
}

\author{
K. Louertani ${ }^{1, *}$, R. Guinvarc' $h^{2}$, N. Ribière-Tharaud ${ }^{3}$, and \\ M. Hélier ${ }^{1}$ \\ ${ }^{1}$ UPMC Univ Paris 06, UR2, L2E, BC 252, Paris 75005, France \\ ${ }^{2}$ Sondra/Supelec, 3 rue Joliot Curie, 91190 Gif-sur-Yvette, France \\ ${ }^{3}$ DRE-L2S at Supelec, 3 rue Joliot Curie, 91190 Gif-sur-Yvette, France
}

\begin{abstract}
This paper deals with an investigation on the polarization of an antenna array with a circular geometry. The theory shows that the polarization of a circular array exhibits a circular polarization independent of the antenna elements. Circular polarization is then achieved whatever the polarization of the elements of the array. Moreover, due to the circular geometry of the array, the entire far field pattern of the array can be obtained with the measurement or the simulation of only one antenna inside the array. The influence of the array radius on the polarization performances has been investigated. An antenna array has been built, and measurements have been performed in order to corroborate the theoretical results.
\end{abstract}

\section{INTRODUCTION}

Circular antenna arrays have been widely investigated in the past to steer the radiation pattern $[1,2]$. Studies have been done to optimize the antenna array characteristics, such as gain, directivity etc. [3-5] and to reduce coupling between elements [6]. In terms of polarization, the antenna array reproduces usually that of its elements. Hence, for an antenna array with a Right Hand Circular Polarization and/or a Left Hand Circular Polarization, the antenna element has to radiate a circular polarization. However, the geometry of the antenna array could also modify the polarization. The investigation in this area [7] shows that a circular polarization can be achieved by

Received 17 June 2011, Accepted 26 September 2011, Scheduled 27 September 2011

* Corresponding author: Karim Louertani (louertani@gmail.com). 
linearly polarized elements in a square or rectangular configuration. In some cases, the antennas are optimized to generate a circular polarization [8]. In this paper, the theory of circular arrays and their influences on the polarization are investigated. An antenna array has been built and measurements have been performed in order to verify that theory. Theoretical and experimental results have shown that a circular polarization can be generated, not only with linearly polarized elements in a square configuration, but whatever the polarization of the antennas composing the array in a circular configuration. The influence of the array radius on the polarization has also been investigated.

\section{ANTENNA ARRAY GEOMETRY}

In order to study the antenna array characteristics, the far field pattern which is the contribution of all the antenna elements, has to be calculated. Thus, the radiation pattern of each element has to be known. The general expression [9] of the radiated electric field is given by the following equation:

$$
\mathbf{E}_{T o t}(\theta, \phi)=\sum_{i=1}^{N} \exp ^{j \Phi_{i}} \mathbf{E}_{i}(\theta, \phi)
$$

where $\mathbf{E}_{i}(\theta, \phi)$ is, in spherical coordinates, the far electric field of element $i$ radiated from where the element is located in the array; $N$ is the number of elements in the antenna array; $\exp ^{j \Phi_{i}}$ is the phase reference of element $i$.

Assuming a weak coupling, the far electric field of element $i$ is expressed, by means of the translational phase-shift theorem, as:

$$
\mathbf{E}_{i}(\theta, \phi)=\mathbf{E}_{0}(\theta, \phi) \exp ^{j k \mathbf{u} \cdot \delta_{i}}
$$

where:

- $k$ is the wavenumber;

- $\mathbf{u}=\sin \theta \cos \phi \mathbf{e}_{x}+\sin \theta \sin \phi \mathbf{e}_{y}+\cos \theta \mathbf{e}_{z} ;$

- $\left(\mathbf{e}_{x}, \mathbf{e}_{y}, \mathbf{e}_{z}\right)$ the usual cartesian basis;

- $\delta_{i}$ the displacement vector of the element from the origin;

- $\mathbf{E}_{0}(\theta, \phi)$ the far electric field radiated when the element is alone and located at the origin.

Thus, all the antenna radiation patterns are taken into account whatever their positions or directions. This study focuses on 2-D antenna arrays with equally spaced elements. More precisely, we investigate circular antenna arrays. Hence, the $N$ elements are placed on a circle of radius $a$. The array geometry has to be periodic (i.e., 
the elements have to be equally spaced). In this configuration, the antennas are arranged in such a way that each antenna geometric configuration results from a rotation of the previous one. For such a configuration, the displacement vector is expressed as:

$$
\delta_{i}=a \cos \left(\frac{2 \pi i}{N}\right) \mathbf{e}_{x}+a \sin \left(\frac{2 \pi i}{N}\right) \mathbf{e}_{y}
$$

Then, with the radiation pattern of only one element, the entire radiation pattern of the antenna array can be calculated. In a more general case, the far electric field in the direction of observation $(\theta, \phi)$ is given by:

$$
\begin{aligned}
\mathbf{E}_{T o t}(\theta, \phi)= & \sum_{i=1}^{N} \mathbf{M}^{-1}(\theta, \phi) \cdot\left(\exp ^{j\left(\frac{2 \pi i}{N}+k \mathbf{u} \cdot \delta_{i}\right)} \mathbf{R}(i, N)\right) \\
& \cdot \mathbf{M}\left(\theta^{\prime}, \phi^{\prime}\right) \mathbf{E}_{0}\left(\theta^{\prime}, \phi^{\prime}\right)
\end{aligned}
$$

where:

- the angles $\left(\theta^{\prime}, \phi^{\prime}\right)$ specify the direction of observation relatively to the array element, in its local spherical coordinate system;

- $\mathbf{E}_{0}\left(\theta^{\prime}, \phi^{\prime}\right)$ is the far electric field of the array element, radiated in the direction $\left(\theta^{\prime}, \phi^{\prime}\right)$, in its local spherical coordinate system;

- $N$ is the number of elements in the antenna array;

- $\exp ^{\left(j \frac{2 \pi i}{N}\right)}$ is the phase shift applied to the $i$ th element;

- the matrix $\mathbf{R}(i, N)$ performs a rotation of $\frac{2 \pi i}{N}$ about the $z$-axis;

- the matrix $\mathbf{M}^{-1}(\theta, \phi)$ performs a transformation from cartesian to global spherical coordinates, after rotation;

- the matrix $\mathbf{M}\left(\theta^{\prime}, \phi^{\prime}\right)$ performs a transformation from local spherical coordinates to cartesian coordinates, before rotation, with:

$$
\begin{aligned}
\mathbf{M}\left(\theta^{\prime}, \phi^{\prime}\right) & =\left(\begin{array}{ccc}
\cos \phi^{\prime} \sin \theta^{\prime} & \cos \phi^{\prime} \cos \theta^{\prime} & -\sin \phi^{\prime} \\
\sin \phi^{\prime} \sin \theta^{\prime} & \sin \phi^{\prime} \cos \theta^{\prime} & \cos \phi^{\prime} \\
\cos \theta^{\prime} & -\sin \theta^{\prime} & 0
\end{array}\right) \\
\mathbf{R}(i, N) & =\left(\begin{array}{ccc}
\cos \left(\frac{2 i \pi}{N}\right) & -\sin \left(\frac{2 i \pi}{N}\right) & 0 \\
\sin \left(\frac{2 i \pi}{N}\right) & \cos \left(\frac{2 i \pi}{N}\right) & 0 \\
0 & 0 & 1
\end{array}\right) \\
\mathbf{M}^{-1}(\theta, \phi) & =\left(\begin{array}{ccc}
\cos \phi \sin \theta & \sin \phi \sin \theta & \cos \theta \\
\cos \phi \cos \theta & \sin \phi \cos \theta & -\sin \theta \\
-\sin \phi & \cos \phi & 0
\end{array}\right)
\end{aligned}
$$


The direction specified by angles $\left(\theta^{\prime}, \phi^{\prime}\right)$ in the local spherical coordinate system of each element is defined, from the global angles $(\theta, \phi)$, by means of the following equation:

$$
\left(\begin{array}{c}
\sin \theta^{\prime} \cos \phi^{\prime} \\
\sin \phi^{\prime} \sin \theta^{\prime} \\
\cos \theta^{\prime}
\end{array}\right)=\left(\begin{array}{ccc}
\cos \left(\frac{-2 i \pi}{N}\right) & -\sin \left(\frac{-2 i \pi}{N}\right) & 0 \\
\sin \left(\frac{-2 i \pi}{N}\right) & \cos \left(\frac{-2 i \pi}{N}\right) & 0 \\
0 & 0 & 1
\end{array}\right)\left(\begin{array}{c}
\sin \theta \cos \phi \\
\sin \phi \sin \theta \\
\cos \theta
\end{array}\right)
$$

It may also be written:

$$
\mathbf{u}^{\prime}=\mathbf{R}(i, N)^{-1} \mathbf{u}
$$

with $\mathbf{u}^{\prime}=\sin \theta^{\prime} \cos \phi^{\prime} \mathbf{e}_{x}+\sin \theta^{\prime} \sin \phi^{\prime} \mathbf{e}_{y}+\cos \theta^{\prime} \mathbf{e}_{z}$.

More details may be given about expression (4). The axis of rotation is always perpendicular to the plane of the antenna array. Hence, since the antenna array is in the plane $x O y$, the rotations of the elements have to be done about $z$-axis. The rotation matrix itself is defined by $\mathbf{R}(i, N)$ in the cartesian coordinate system. The value of the far field of the array element in local direction $\left(\theta^{\prime}, \phi^{\prime}\right)$ is converted from spherical to global cartesian coordinates by matrix $\mathbf{M}\left(\theta^{\prime}, \phi^{\prime}\right)$ and $\left(\theta^{\prime}, \phi^{\prime}\right)$ defined previously. Then, the phase shift due to translation is taken into account, combined with a rotation about $z$-axis. Another conversion is needed from cartesian to global spherical coordinates in direction $(\theta, \phi)$ by means of matrix $\mathbf{M}^{-1}(\theta, \phi)$.

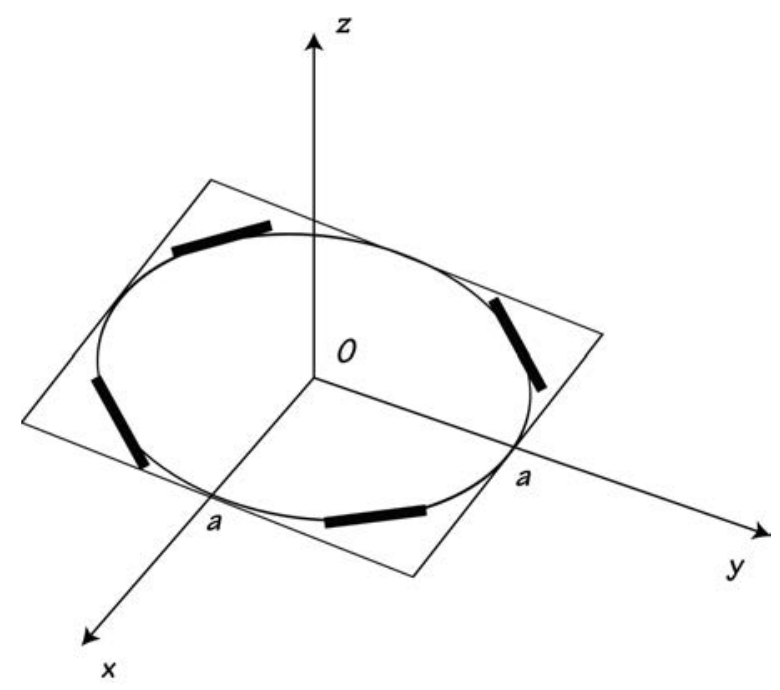

Figure 1. 3D view of the antenna array geometry. 
Moreover, a phase shift is applied to each element by multiplying the result by $\exp ^{j \frac{2 \pi i}{N}}$. This operation is done $N$ times and the contributions are summed in order to obtain the radiation pattern of the whole antenna array.

An example is given in Figure 1, where a black segment depicts an antenna element (horizontal short dipole). In this example, four antennas are arranged along a circle in the plane $x O y$ and it can be seen that these array elements are obtained from rotations about the center of $45^{\circ}, 135^{\circ}, 225^{\circ}$ and $315^{\circ}$ of an original element, supposed to be a $y$-oriented, horizontal short dipole located at point $(a, 0,0)$.

Due to the rotational symmetry, coupling between the elements can be considered by assuming that $\mathbf{E}_{i}(\theta, \phi)$ is calculated or measured with all the elements. In practice, this means that $\mathbf{E}_{i}(\theta, \phi)$ is obtained by feeding only an antenna element in the antenna array. Thus, coupling is included in the radiation pattern of the antenna reference. In that case, Equation (1) should be directly applied with $\mathbf{E}_{i}(\theta, \phi)$, the radiated far electric field of element $i$, located in its actual environment, which includes coupling effects.

\section{POLARIZATION OF THE CIRCULAR ANTENNA ARRAY}

For a classical linear antenna array, the polarization depends on that of the antenna element. Thus, if the antenna array is composed of linear polarized elements, the polarization of the array will be linear. In this part, the influence of the antenna array geometry is studied. For simplicity, the study focuses on the direction defined by $\theta=0^{\circ}$ and $\phi=0^{\circ}$. In this direction, the total far electric field becomes:

$$
\mathbf{E}_{\text {Tot }}(0,0)=\sum_{i=1}^{N}\left(\begin{array}{ccc}
1 & 0 & 0 \\
0 & \cos \left(\frac{2 \pi i}{N}\right) & \sin \left(\frac{2 \pi i}{N}\right) \\
0 & -\sin \left(\frac{2 \pi i}{N}\right) & \cos \left(\frac{2 \pi i}{N}\right)
\end{array}\right) \exp ^{\left(j \frac{2 \pi i}{N}\right)} \mathbf{E}_{i}(0,0)
$$

Let us assume that $\mathbf{E}_{\text {Tot }}(\theta, \phi)=E_{\theta_{T o t}}(\theta, \phi) \mathbf{e}_{\theta}+E_{\phi_{T o t}}(\theta, \phi) \mathbf{e}_{\phi}$. To achieve circular polarization, $E_{\theta_{T o t}}(\theta, \phi)$ and $E_{\phi_{T o t}}(\theta, \phi)$ have to satisfy the following conditions:

$$
\left\{\begin{array}{l}
\left|E_{\theta_{T o t}}\right|=\left|E_{\phi_{T o t}}\right| \\
\arg \left(E_{\theta_{T o t}}\right)-\arg \left(E_{\phi_{T o t}}\right)= \pm \pi / 2
\end{array}\right.
$$

Table 1 gives the phase shift with formulas of $E_{\theta_{T o t}}$ and $E_{\phi_{T o t}}$ according to the number of elements $N$ in the array. For the first two cases, which correspond to only one or two elements, the values of $E_{\theta_{T o t}}$ and $E_{\phi_{T o t}}$ depend on the antenna element because the array is 
Table 1. $E_{\theta_{T o t}}$ and $E_{\phi_{T o t}}$ according to the number of elements $N$.

\begin{tabular}{|c|c|c|c|c|}
\hline$N$ & 1 & 2 & 3 & 4 \\
\hline$E_{\theta_{T o t}}(\theta=0, \phi=0)$ & $E_{\theta}$ & $2 \times E_{\theta}$ & $\frac{3}{2} \times\left(E_{\theta}+j E_{\phi}\right)$ & $2 \times\left(E_{\theta}+j E_{\phi}\right)$ \\
\hline$E_{\phi_{T o t}}(\theta=0, \phi=0)$ & $E_{\phi}$ & $2 \times E_{\phi}$ & $\frac{3}{2} \times\left(-j E_{\theta}+E_{\phi}\right)$ & $2 \times\left(-j E_{\theta}+E_{\phi}\right)$ \\
\hline $\arg \left(E_{\theta_{T o t}}\right)-\arg \left(E_{\phi_{T o t}}\right)$ & $x$ & $x$ & $\pm \pi / 2$ & $\pm \pi / 2$ \\
\hline
\end{tabular}

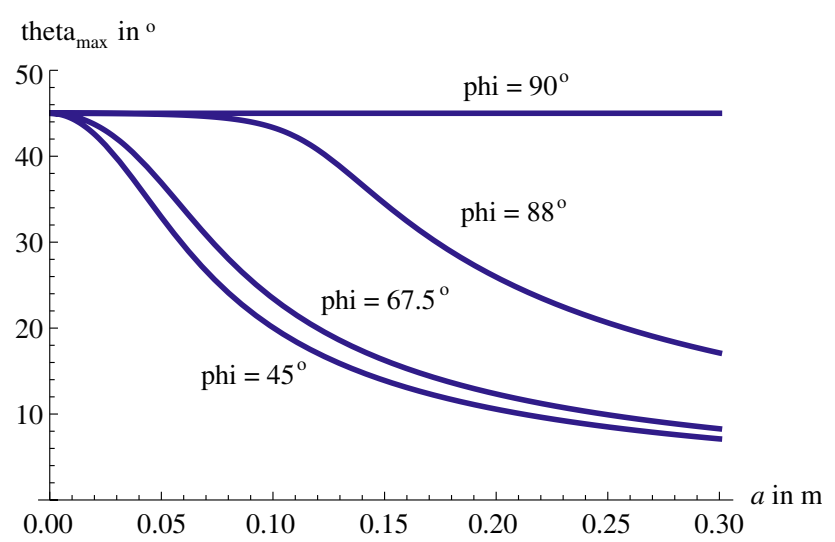

Figure 2. Theta max in degree corresponding to an $\mathrm{AR} \geq-3 \mathrm{~dB}$ according to the radius ' $a$ ' for $\phi=45^{\circ}, 67.5^{\circ}, 88^{\circ}$ and $90^{\circ}$.

linear. Thus, the radiation pattern and the polarization are those of the generating element.

From $N=3$, the components of the far field can be written as in (12) and $E_{\theta_{T o t}}$ and $E_{\phi_{T o t}}$ completely satisfy the conditions given in (11). Hence, for a minimum of 3 elements, the antenna array is perfectly circular polarized, in the direction $\theta=0^{\circ}$ and $\phi=0^{\circ}$, whatever the polarization of the antenna element.

$$
\left\{\begin{array}{l}
E_{\theta_{T o t}}=\frac{N}{2} \times\left(E_{\theta}+j E_{\phi}\right) \\
E_{\phi_{T o t}}=\frac{N}{2} \times\left(-j E_{\theta}+E_{\phi}\right)
\end{array}\right.
$$

In a way to perform a circular polarization over a wide angular range, $E_{\theta_{T o t}}$ and $E_{\phi_{T o t}}$ have to satisfy the condition given in (11) over an angular range as wide as possible. This depends on the antenna element characteristics and especially on its radiation pattern. Indeed, the radiation pattern has to be as large as possible in order to cover a wide angular range. Moreover, the radius ' $a$ ' should not be too large to minimize the phase delay between elements in order to maintain an acceptable angular coverage of circular polarization. Figure 2 shows the maximum value of $\theta$ corresponding to an $\mathrm{AR} \geq-3 \mathrm{~dB}$ according 
to the radius ' $a$ ' for $\phi=45^{\circ}, 67.5^{\circ}, 88^{\circ}$ and $90^{\circ}$. The simulations have been performed at the frequency $1 \mathrm{GHz}(\lambda=30 \mathrm{~cm})$. The radius ' $a$ ' influences the angular coverage of circular polarization. The coverage is maximum when the elements are close to each others, i.e., ' $a$ ' close to 0 , and minimum when the distance increases. The angle $\phi=90^{\circ}$ is a particular solution that exhibits a constant angular coverage for different values of radius.

\section{LPDA ANTENNAS ARRAYS}

In this section, the theory is applied to a Log Periodic Dipole Array antenna (LPDA). The LPDA is used as an antenna element in a circular

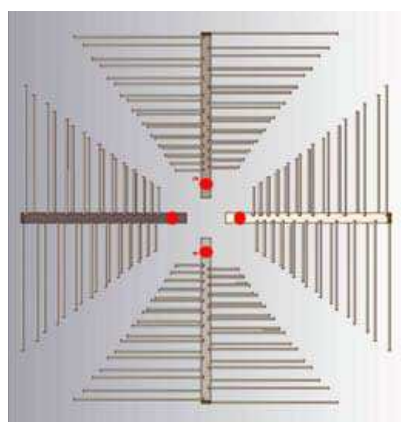

(a)

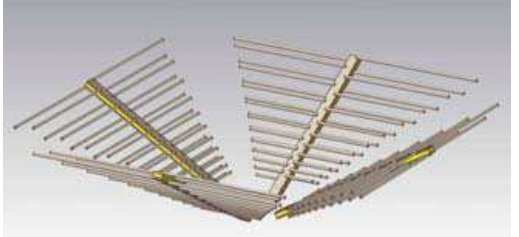

(b)

Figure 3. (a) Top view and (b) $3 \mathrm{D}$ view of the $4 \times$ LPDA antennas modeled with CST microwave studio.

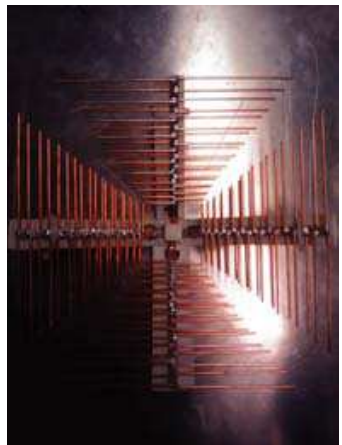

(a)

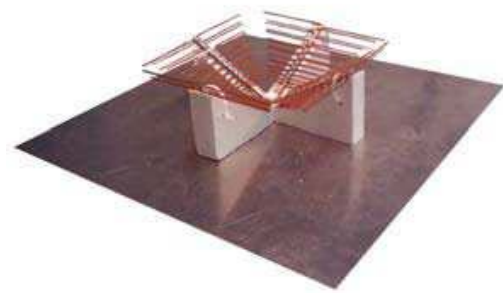

(b)

Figure 4. (a) Top view and (b) $3 \mathrm{D}$ view of the $4 \times$ LPDA antennas. 
array of four elements. The antenna array has been modeled using the

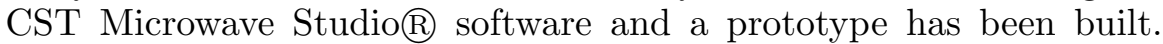
Firstly, simulation and measurement results of an antenna element

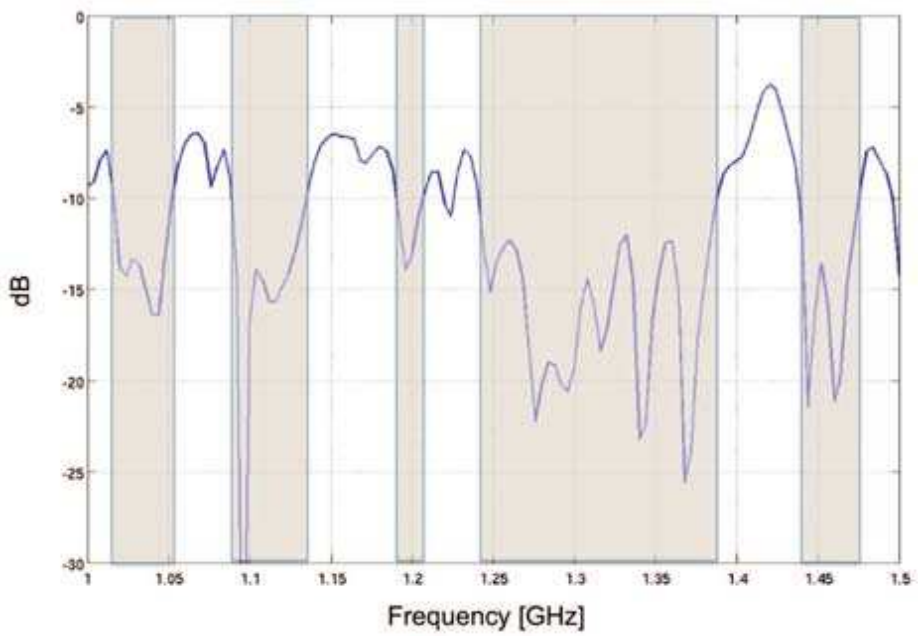

Figure 5. Measured $\left|S_{11}\right|$ of an LPDA antenna.

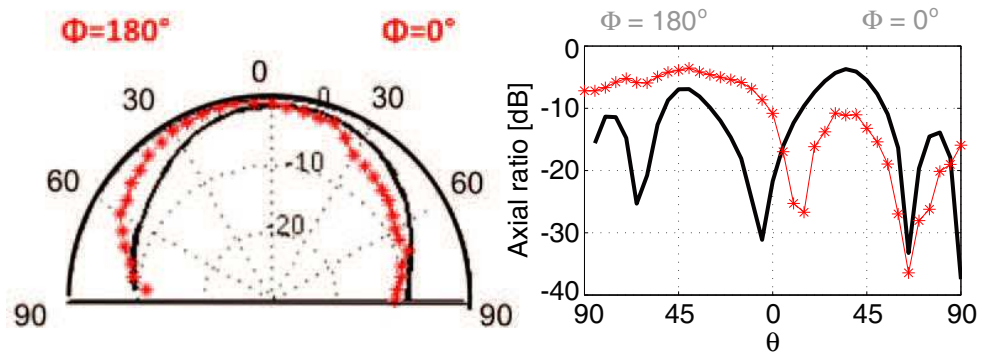

(a)
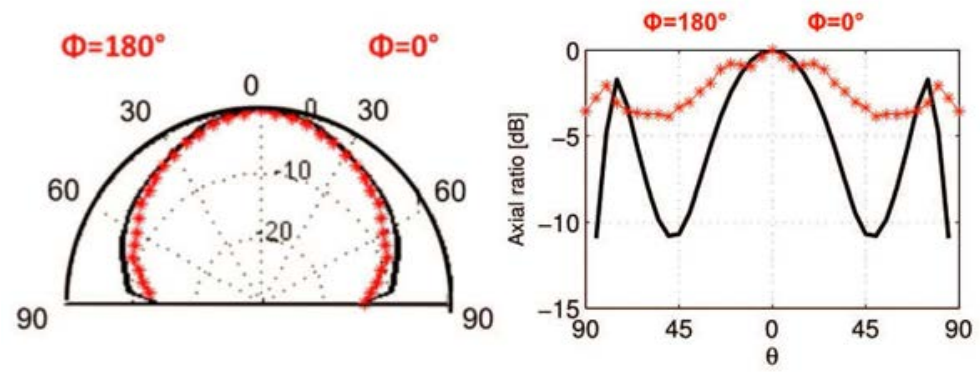

(b) 

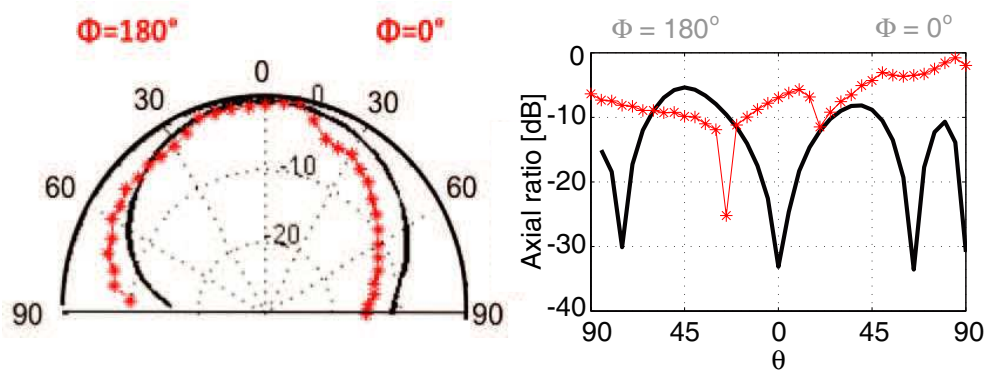

(c)

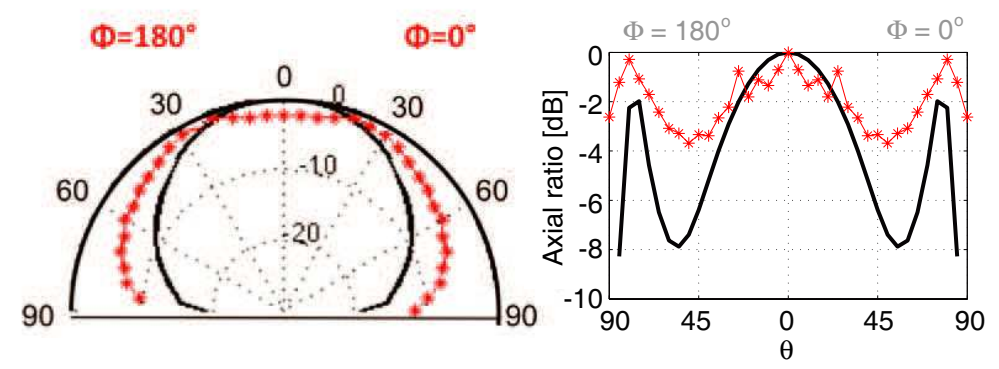

(d)

Figure 6. Measured and simulated cuts in elevation of the normalized total gain (left) and AR (right) of an antenna element and an antenna array at (a) (b) $1 \mathrm{GHz}$ and (c) (d) $1.1 \mathrm{GHz}$. (a) Normalized total gain (left) and AR (right) of an element at $1 \mathrm{GHz}$. (b) Normalized total gain (left) and AR (right) of the array at $1 \mathrm{GHz}$. (c) Normalized total gain (left) and AR (right) of an element at 1.1 GHz. (d) Normalized total gain (left) and AR (right) of the array at $1.1 \mathrm{GHz}$.

inside the array are compared. Secondly, the far field pattern of the antenna array calculated with the measurement results, according to the theory, is compared with the simulated array.

In this case, four elements are placed along a circle and a phase shift of $90^{\circ}$ is applied between each antenna. The LPDA [10] antenna is built by assembling 21 dipoles for a frequency range between $1 \mathrm{GHz}$ and $2 \mathrm{GHz}$. The modeled antenna array is presented in Figure 3 and the prototype in Figure 4. The array is placed at the center of a $40 \mathrm{~cm} \times 40 \mathrm{~cm}$ ground plane.

The $\left|S_{11}\right|$ coefficient of an antenna element is plotted in Figure 5 for the frequency range from $1 \mathrm{GHz}$ to $1.5 \mathrm{GHz}$. The antenna radiates over several frequency bands marked by grayed surfaces where the absolute value of the reflexion coefficient is under $-10 \mathrm{~dB} .\left|S_{11}\right|$ 
is almost always lower than $-7 \mathrm{~dB}$ over the remaining bandwidth. Measurement and simulation results of both an element and the array are compared in Figure 6 at frequencies of $1 \mathrm{GHz}$ and $1.1 \mathrm{GHz}$. In this figure, cuts in elevation of the normalized total gain and the axial ratio (AR) are represented. The red cross denotes the measurement results while the black lines show the simulation ones. It is clear that measurement results are in good agreement with simulation. The antenna element radiates a main beam in the perpendicular direction of the ground plane and, as expected, the polarization is not circular. Indeed, simulated $\mathrm{AR}$ is lower than $-20 \mathrm{~dB}$ at $\theta=0^{\circ}$. The radiation pattern and the AR of the antenna array are calculated, according to the theory, from the measurement of an antenna element included in the array. The total gain exhibits a main symmetrical beam on the perpendicular axis of the ground plane. According to the theory, the polarization is perfectly circular in the boresight for $\theta=0^{\circ}$ and the $\mathrm{AR}$ is greater than $-3 \mathrm{~dB}$ over a wide angular range. Measured polarization shows better results than simulation. This is probably due to an imperfect symmetry of the LPDA on the ground plane.

\section{CONCLUSION}

In this paper, the idea of using circular array to generate a circular polarization, whatever the elements of the array, has been presented. By using only the measurement of an antenna element inside the array, the entire far field pattern including coupling between elements could be calculated. According to the theory and the measurement, a perfect circular polarization is achieved in the boresight and an AR $\geq-3 \mathrm{~dB}$ over an angular range depending on the antenna element. The array radius influences the performances of the radiated polarization. Simulations have shown that the angular coverage of the circular polarization decreases when the radius increases. An antenna array of $4 \times$ LPDA has been built and measured. Good agreements have been observed between simulated and measured results. The antenna array can be optimized to find the best compromise between the coupling effects and an angular coverage with a good circular polarization.

\section{REFERENCES}

1. Neff, H. and J. Tillman, "An electronically scanned circular antenna array," Proc. IRE International Convention Record, Vol. 8, 41-47, Mar. 1960. 
2. Simpson, T. and J. Tillman, "Parasitic excitation of circular antenna arrays," IEEE Trans. Antennas and Propagat., Vol. 9, No. 3, 263-267, May 1961.

3. Shihab, M., Y. Najjar, N. Dib, and M Khodier, "Design of non-uniform circular antenna arrays using particle swarm optimization," Electrical Engineering, Vol. 59, 216-220, 2008.

4. Gurel, L. and O. Ergul, "Design and simulation of circular arrays of trapezoidal-tooth log-periodic antennas via genetic optimization," Progress In Electromagnetics Research, Vol. 85, 243-260, 2008.

5. Chang, T.-N. and J.-H. Jiang, "Enhance gain and bandwidth of circularly polarized microstrip patch antenna using gap-coupled method," Progress In Electromagnetics Research, Vol. 96, 127139, 2009.

6. Davies, D. E. N., "Correspondence: Mutual coupling compensation for small, circularly symmetric planar antenna arrays," IEE Proceedings H Microwaves, Optics and Antennas, Vol. 129, No. 5, 281-283, Oct. 1982.

7. Huang, J., "A technique for an array to generate circular polarization with linearly polarized elements," IEEE Trans. Antennas and Propagat., Vol. 34, No. 9, 1113-1124, Sep. 1986.

8. Herscovici, N., Z. Sipus, and D. Bonefacic, "Circularly polarized single-fed wide-band microstrip patch," IEEE Trans. Antennas and Propagat., Vol. 51, No. 6, 1277-1280, Jun. 2003.

9. Chauvet, F., R. Guinvarc'h, and M. Hélier, "Approximated method neglecting coupling for conformal array," Applied Computational Electromagnetics Society Journal, Vol. 22, 105111, Mar. 2007.

10. Guinvarc'h, R. and N. Ribière-Tharaud, "A wideband antenna for P-band airborne SAR applications," IEEE Antennas and Propagation Society International Symposium, AP-S, 1-4, 2008. 